CNS Spectrums (2016), 21, 9-11. C Cambridge University Press 2015. The online version of this article is published within an Open Access environment subject to the conditions of the Creative Commons Attribution-NonCommercial-ShareAlike licence <http://creativecommons.org/licenses/by-nc-sa/3.0/ > . The written permission of Cambridge University Press must be obtained for commercial re-use.

doi:10.1017/S1092852915000541

First published online 4 November 2015

\title{
Hypoactive Sexual Desire Disorder: Separating Fact from Fiction
}

\author{
Anita H. Clayton ${ }^{\prime *}$ and Dennis H. Kim ${ }^{2}$
}

\author{
${ }^{1}$ Department of Psychiatry and Neurobehavioral Sciences, University of Virginia, Charlottesville, Virginia, USA \\ ${ }^{2}$ Arbor Scientia, Carlsbad, California, USA
}

Hypoactive sexual desire disorder (HSDD) and its potential treatments have received high-profile media coverage (a notable recent example was "The Pink Pill: Viagra for the Female Brain," presented by Katie Couric for Yahoo! News). ${ }^{1}$ Throughout this public discourse, critics have expressed sentiments ranging from skepticism to categorical opposition with respect to both the disorder itself as well as its developmental pharmacotherapies in general (and flibanserin in particular). The criticisms leveled toward HSDD have been based on several misconceptions that we felt warranted a response based on published science.

\section{Are Female Sexual Problems Real?}

This is a question that has long been central to debates over women's sexual rights. Some argue that the absence of spontaneous or responsive desire does not signal a pathology. Others suggest that female sexual disorders, specifically HSDD, are simply a conspiracy perpetrated by the pharmaceutical industry to pathologize what is within the spectrum of normal sexual behavior in order to mislead women to feel that their sexual function is inadequate and that their problems are simple and easy to treat with drugs. However, women who are satisfied with their level of sexual desire, or who are not distressed by a low level of sexual desire, do not seek intervention of any kind, and so would not succumb to such promotions. Additionally, these viewpoints blithely dismiss what we know about the biology of sexual desire and arousal, as well as the clinical consensus of healthcare providers. The current absence of a definitive mechanism for HSDD should not be mistaken for an absence of empirical data and evidence of

* Address for correspondence: Anita H. Clayton, MD, Department of Psychiatry and Neurobehavioral Sciences, University of Virginia, 2955 Ivy Road, Northridge Suite 210, Charlottesville, VA 22903, USA. (Email: ahc8v@virginia.edu) neurobiological mechanisms underlying both sexual function and dysfunction. It is well accepted that the modulation of sexual desire is impacted by numerous neuroendocrine entities, including hormones (estrogen, androgens, melanocortins, oxytocin, prolactin) and neurotransmitters (dopamine, norepinephrine, and serotonin). ${ }^{2,3}$ For example, sexual excitation involves sexual cues activating hypothalamic and limbic norepinephrine and oxytocin (which in turn stimulate sexual arousal) and dopamine and melanocortins (which stimulate attention and desire). ${ }^{2}$ On the flipside of this mechanism, but relatively less well understood, is the inhibitory influence exerted by brain opioid, endocannabinoid, and serotonin systems acting in several regions, including the prefrontal cortex (PFC). ${ }^{2}$ In fact, multiple neuroimaging studies of women with and without HSDD have shown that a woman's prefrontal cortex plays a central role in regulating sexual function. ${ }^{4,5}$ In women with HSDD, the dysregulation of serotonin and dopamine in the PFC is believed to overly inhibit the sexual response. This topdown phenomenon is just one type of dysfunction that signals the possibility of a malfunction in reward circuitry.

Although critics are correct to observe that low sexual desire may result from a number of medical conditions (eg, major depressive disorder, diabetes), medications (eg, antidepressants), and interpersonal/psychosocial factors (eg, relationship problems), it is a fallacy to assume that the existence of these etiologies precludes others. ${ }^{6}$ To take the example of antidepressants, their well-known potential to decrease libido, in fact, has made it clear that alterations in monoamine neurotransmission can have significant negative effects on sexual desire. In any event, this line of argument simply highlights the fact that accurate and appropriate diagnosis of HSDD is not a trivial matter. It is vital that healthcare providers receive education on how to make or exclude the diagnosis of HSDD and how to 
appropriately prescribe treatments, when they become available; the Decreased Sexual Desire Screener (DSDS) can be a useful tool in this process. ${ }^{7}$ Given these possibilities, the development and safe use of therapeutic interventions for the disorder should not be abandoned.

A second line of argument against HSDD has involved nosological changes brought about by the release of the Diagnostic and Statistical Manual of Mental Disorders, Fifth Edition (DSM-5). At least one vocal opponent has questioned the legitimacy of HSDD by pointing out that the diagnosis no longer exists in the DSM-5, thus claiming that HSDD is therefore no longer an actual medical condition. ${ }^{1}$ This argument is false and misleading. The DSM-5 now includes female sexual interest/ arousal disorder (FSIAD), which subsumes the diagnostic criteria of both HSDD and female sexual arousal disorder (FSAD) from the Diagnostic and Statistical Manual of Mental Disorders, 4th Edition, Text Revision (DSM-IV-TR). ${ }^{8}$ In recent years, several of my colleagues and I have argued vigorously against the merging of HSDD and FSAD into the combined diagnosis. In fact, at an October 2014 meeting held by the U.S. Food and Drug Administration (FDA) to discuss female sexual dysfunction, the changes made in the DSM-5 were widely criticized by several leaders in the fields of sexual medicine, gynecology, and psychiatry. The objections to the changes in DSM-5 have been rooted in (1) the knowledge that the dysfunctions of desire and objective arousal are mediated by different biological factors, and (2) evidence that premenopausal women with HSDD and women with FSAD according to DSM-IV-TR have distinct symptom patterns. ${ }^{9,10}$ Surprisingly, there are no published clinical studies to validate the FSIAD diagnosis in the general population or in women presenting with symptoms of sexual dysfunction (clinical population). Tabling this debate for the time being, the fact remains that whether termed HSDD or FSIAD, the characteristic symptomatology associated with a "lack of, or significantly reduced, sexual interest" and its resultant clinically significant distress continue to be recognized by the American Psychiatric Association as a legitimate disorder. Furthermore, the International Classification of Diseases (ICD) codes, used by almost all clinicians for diagnosis and billing, continue to include female HSDD. ${ }^{11,12}$

As a final comment, the authors do not feel that the pursuit of safe and effective pharmacotherapies for HSDD/FSIAD invalidates or in any way runs counter to the application of other, nonpharmacological tools in the treatment provider's armamentarium. Indeed, any treatment regimen would be remiss if it did not fully explore and take into account somatic, psychological, and psychosocial factors in the patient's life. Unfortunately, randomized controlled trials of psychotherapy interventions for HSDD have not demonstrated superiority to a control paradigm comparable to placebo. Even the psychotherapies that appear to be more effective than a wait-list control condition (not equivalent to placebo) are not effective in all women. So, just as there is no single profile of sexual function, there is no single therapeutic option that is guaranteed to work for all patients. We strongly feel that having more approved therapeutic options available ultimately serves the interests and needs of patients for whom other avenues have proven to be inadequate.

\section{Disclosures}

Anita Clayton has the following disclosures: in the last 12 months, Dr. Clayton has received grants from Auspex Pharmaceuticals, Forest Research Institute, Inc., Palatin Technologies, and Trimel Biopharma; advisory board fees/consultant fees from Arbor Scientia, Forest Labs, Lundbeck, Naurex, Otsuka, Palatin Technologies, Pfizer, Inc., Roche, S1 Biopharmaceuticals, Sprout Pharmaceuticals, and Takeda Global Research \& Development; royalties/copyright from Ballantine Books/Random House, Changes in Sexual Functioning Questionnaire, Guilford Publications; and shares/restricted stock units in Euthymics, S1 Biopharmaceuticals, Inc. Dennis Kim is an employee of Arbor Scientia, a consultant of Sprout Pharmaceuticals.

\section{REFERENCES:}

1. Couric K. The pink pill: viagra for the female brain. Yahoo! News. http://news.yahoo.com/katie-couric-little-pink-pill-and-the-drugsresubmission-for-fda-approval-031600521.html. Accessed August 19, 2015.

2. Pfaus JG. Pathways of sexual desire. J Sex Med. 2009; 6(6): 1506-1533.

3. Nappi RE, Martini E, Terreno E, et al. Management of hypoactive sexual desire disorder in women: current and emerging therapies. Int J Womens Health. 2010; 2: 167-175.

4. Bianchi-Demicheli F, Cojan Y, Waber L, Recordon N, Vuilleumier P, Ortigue $\mathrm{S}$. Neural bases of hypoactive sexual desire disorder in women: an event-related fMRI study. J Sex Med. 2011; 8(9): 2546-2559.

5. Woodard TL, Nowak NT, Balon R, Tancer M, Diamond MP. Brain activation patterns in women with acquired hypoactive sexual desire disorder and women with normal sexual function: a cross-sectional pilot study. Fertil Steril. 2013; 100(4): 1068-1076.

6. Clayton AH. The pathophysiology of hypoactive sexual desire disorder in women. Int J Gynaecol Obstet. 2010; 110(1): 7-11.

7. Clayton AH, Goldfischer ER, Goldstein I, DeRogatis L, Lewis-D'Agostino DJ, Pyke R. Validation of the Decreased Sexual Desire Screener (DSDS): a brief diagnostic instrument for generalized acquired female hypoactive sexual desire disorder (HSDD). J Sex Med. 2009; 6(3): 730-738.

8. American Psychiatric Association. Diagnostic and Statistical Manual of Mental Disorders. 5th ed. Arlington, VA: American Psychiatric Association; 2013.

9. DeRogatis LR, Clayton AH, Rosen RC, Sand M, Pyke RE. Should sexual desire and arousal disorders in women be merged? Arch Sex Behav. 2011; 40(2): 217-219. 
10. Clayton AH, DeRogatis LR, Rosen RC, Pyke R. Intended or unintended consequences? The likely implications of raising the bar for sexual dysfunction diagnosis in the proposed DSM-V revisions: 1 . For women with incomplete loss of desire or sexual receptivity. J Sex Med. 2012; 9(8): 2027-2039.
11. 2014 ICD-9-CM Diagnosis Code 302.71: Hypoactive sexual desire disorder. http://www.icd9data.com/2014/Volume1/290-319/300316/302/302.71.htm.

12. 2015 ICD-10-CM Diagnosis Code F52.0: Hypoactive sexual desire disorder. http://www.icd10data.com/ICD10CM/Codes/F01-F99/ F50-F59/F52-/F52.0. 